\title{
Barreras para la práctica de ejercicio físico en universitarios mexicanos comparaciones por género Barriers for practicing physical exercise in Mexican university students: gender comparisons José René Blanco Ornelas, Ma. Concepción Soto Valenzuela, Zuliana Paola Benitez Hernández, Fernando Mondaca Fernández, Perla Jannet Jurado García UniversidadAutónoma de Chihuahua (México)
}

Resumen. El objetivo de esta investigación consistió comparar los perfiles de barreras para la práctica de ejercicio físico de estudiantes de universitarios mexicanos. La muestra total fue de 897 participantes, 490 mujeres y 407 estudiantes universitarios de la ciudad de Chihuahua, México, con edades entre los 18 y 36 años. El abordaje adoptado en la investigación se enmarcó dentro de un enfoque cuantitativo con un diseño descriptivo tipo encuesta. Se utilizó un análisis de varianza múltiple (MANOVA) para examinar las diferencias entre mujeres y hombres universitarios en cuanto a la percepción de barreras para la práctica de ejercicio físico. Los resultados del MANOVA mostraron diferencias globales estadísticamente significativas de acuerdo a la variable género en las puntuaciones promedio de los factores del Autoinforme de Barreras para la Práctica de Ejercicio Físico (Wilks’ ë = .884; $p<.001$ ). Posteriormente, los resultados de los ANOVAs mostraron que las mujeres reportan mayor presencia de barreras en las cuatro dimensiones estudiadas; ansiedad física y social $(F=40.281, p<.001)$, fatiga o pereza $(F=94.004, p<.001)$, obligaciones y falta de tiempo $(F=66.774, p<.001)$ e instalaciones $(F=20.925, p<.01)$. Las diferencias encontradas parecen indicar que las mujeres perciben mayor probabilidad en la presencia de barreras para la práctica de ejercicio físico, dificultándoles la práctica de actividad física en mayor medida que a los hombres. Palabras clave: actividad física, diferencias de género, educación superior, barreras, autopercepciones.

\begin{abstract}
The aim of this research was to compare the profiles of barriers for practicing physical exercise in Mexican university students. The total sample was composed by 897 subjects, 490 female and 407 male university students from Chihuahua, Mexico, with a mean age of 18 and 36 years old. The research is framed within a quantitative approach with a survey descriptive design. A multivariate analysis of variance (MANOVA) was used to examine the differences between university men and women regarding the perception of barriers for practicing physical exercise. Results from the MANOVA showed statistically significant global differences according to gender in the average factor scores of the Self-Report of Barriers for Practicing Physical Exercise (Wilks' ë $=.884 ; \mathrm{p}<.001$ ). Subsequently, the results of the ANOVAs showed that women report a greater presence of barriers in the four dimensions studied; physical and social anxiety ( $F=40.281, p<.001)$, fatigue or laziness $(F=94.004$, $p<.001)$, obligations and lack of time $(F=66.774, p<.001)$ and facilities $(F=20.925, p<.01)$. The encountered differences seem to indicate that women have greater probability of perceiving barriers for the practice of physical exercise, making it more difficult for them to practice physical activity than for men.
\end{abstract}

Keywords: physical activity, gender differences, higher education, barriers, self-perceptions.

\section{Introducción}

El sedentarismo es un factor de riesgo a nivel mundial relacionado con enfermedades crónicas no transmisibles y con muertes prematuras (OMS, 2018; Vélez-Álvarez, Vidarte-Claros, \& Parra-Sánchez, 2014); además existen otros factores de riesgo como la modernización, las dietas inadecuadas, el estrés, el tabaquismo y el consumo de alcohol y drogas (Paredes, Orraca, Marimón, Casanova, \& González, 2014; Velasco-Contreras, 2013), incluso la tecnología ha propiciado cambios para ser más sedentarios, con ejercicios breves y ocasionales a una intensidad moderada o vigorosa durante el día (Muñoz-Daw, MuñozDuarte, De La Torre-Díaz, Hinojos-Seáñez, \& Pardo-Rentería, 2016).

Sin embargo, a pesar de las múltiples evidencias que conllevan los beneficios de la actividad física, la mayoría de la población permanece sedentaria y durante su tiempo libre no practican ningún tipo de actividad física (Niñerola, Capdevila, \& Pintanel, 2006). Si las personas sedentarias realizaran actividad física constante disminuirían su mortalidad, así como el riesgo de muerte por problemas cardiovasculares (OMS, 2018).

Desafortunadamente uno de cada cuatro adultos no tiene un nivel suficiente de actividad física, y más del $80 \%$ de la población adolescente no realiza la actividad física necesaria que le permita obtener beneficios sobre la salud(OMS, 2018). En México, la Encuesta Nacional de Salud y Nutrición (ENSANUT, 2012) informa que más de la mitad de los adolescentes no realizan actividad física regular y de los adultos solo el 26.1\% realiza actividad física frecuentemente (Gutiérrez et al., 2012).

Por otra parte, de acuerdo con Niñerola et al. (2006) las barreras para la práctica de la actividad física han ido aumentado en países desarrollados debido a diferentes factores como la dependencia de la tecnología, la urbanización de la población y el ámbito laboral. Entre las barreras más comunes se encuentran la falta de tiempo disponible y las obligaciones laborales o familiares, sobre todo en las mujeres (Juarbe,

Fecha recepción: 04-10-18. Fecha de aceptación: 11-01-19 Perla Jannet Jurado García

pjurado@uach.mx
Turok, \& Perez-Stable, 2002), otras barreras reportadas son la falta de instalaciones deportivas, la falta de interés, la falta de dinero, experiencias negativas, entre otras (OMS, 2018; Ramírez-Vélez, Triana-Reina, Carrillo, \& Ramos-Sepúlveda, 2016).

Las alteraciones negativas tales como las metabólicas y las nutricionales contribuyen también a estas barreras para cumplir con la práctica de ejercicio físico (Stankov, Olds, \& Cargo, 2012), lo que se relaciona con padecer riesgo de sobrepeso, obesidad, diabetes y otras enfermedades no transmisibles (Fisher et al., 2016; Leone \& Ward, 2013).

Algunas de las barreras en las mujeres con obesidad se relacionan con una mala percepción de su autoconcepto total, físico y emocional (Meza-Peña \& Pompa-Guajardo, 2016).

Así pues, la inactividad física y la obesidad se explican en parte debido a barreras internas o autopercibidas y a barreras externas como el acceso, el entorno y la infraestructura; las cuales impiden que el sujeto adopte un estilo de vida saludable (Ramírez-Vélez et al., 2015; RamírezVélez et al., 2016).

En relación a los estudiantes universitarios, son varios los autores que afirman que las barreras externas hacia la práctica de ejercicio físico están asociadas con la falta de tiempo, de instalaciones cercanas y de apoyo social, y entre las barreras internas se encuentran la falta de interés por la actividad física, el no encontrarle utilidad, sentirse flojo, apático o incompetente para practicar actividad física, falta de recursos y miedo a lastimarse (El-Gilany, Badawi, El-Khawaga, \& Awadalla, 2011; Gómez-López, Gallegos, \& Extremera, 2010; Lovell, Ansari, \& Parker, 2010; Patiño-Villada, Arango-Vélez, Quintero-Velásquez, \& Arenas-Sosa, 2011; Ramírez-Vélez et al., 2015; Serra, Generelo, \& Zaragoza, 2010).

Por otro lado, en relación al género, se ha encontrado que existe una mayor proporción en los niveles de actividad física bajo en las mujeres en comparación con los hombres (Arboleda, Arango, \& Feito, 2016; Meneses \& Ruiz, 2017). De acuerdo a un estudio realizado por RamírezVélez et al. (2016) entre las barreras para la práctica de actividad física tales como: falta de tiempo, influencia social, falta de energía, falta de voluntad, miedo a lastimarse, falta de habilidades y falta de recursos, las 
principales barreras fueron la falta de tiempo y la falta de recursos, siendo las mujeres quienes mostraron niveles más altos en cada una de las barreras. Además, Pavón y Moreno (2008) realizaron una investigación con 1512 estudiantes de la Universidad de Murcia sobre las motivaciones e intereses relacionados con la práctica físico deportiva, encontrando que las mujeres son más sedentarias porque no les gusta la competencia y los objetivos a largo plazo que dificultan el mantenimiento de la práctica de ejercicio, mientras que, los varones por el contrario, disfrutan el ejercicio y la competición, recibiendo un refuerzo inmediato que mantiene su conducta.

En este sentido, cobra especial relevancia indagar si pertenecer a determinado género contribuye o no a percibir con mayor probabilidad la presencia de barreras para la práctica de ejercicio físico, dificultando la práctica de esta.

Por tanto, el presente estudio intenta determinar las diferencias y similitudes entre hombres y mujeres universitarios mexicanos en cuanto a la percepción de barreras para la práctica de ejercicio físico.

En consecuencia, esta investigación pretende, como investigación aplicada, aportar información que se traduzca en una práctica educativa de mayor calidad en el contexto de atención a la diversidad; contribuyendo al saber pedagógico en el esclarecimiento de los factores que conforman un modelo de desarrollo humano integral.

\section{Metodología}

\section{Participantes}

Participaron en el estudio 897 sujetos, 490 mujeres y 407 hombres todos alumnos de la Facultad de Ciencias de la Cultura Física (FCCF) de la UniversidadAutónoma de Chihuahua, México. La edad de los sujetos fluctuó entre los 18 y 36 años, con una media de 20.84 y una desviación estándar de 2.54 años.

La muestra se obtuvo mediante un muestreo por conveniencia, tratando de abarcar la representatividad de los diferentes semestres de las licenciaturas que se ofrecen en la FCCF.

\section{Instrumento}

El Autoinforme de Barreras para la Práctica de Ejercicio Físico (ABPEF) de Niñerola et al. (2006) consta de 17 ítems, agrupados en cuatro factores (ansiedad física y social, fatiga o pereza, obligaciones y falta de tiempo e instalaciones), que se responden según una escala tipo Likert de 0 a 10 puntos, donde valores cercanos a 0 indican «una razón poco probable que me impide practicar ejercicio físico las próximas semanas», y valores cercanos a 10 indican una «razón muy probable que me impide practicar ejercicio físico». Para nuestro estudio se hicieron dos adaptaciones a la versión de Niñerola et al. (2006): (a) la primera consistió en cambiar algunos términos utilizados en los ítems de la versión original con el fin de emplear un lenguaje más adecuado al contexto de la cultura mexicana; (b) la segunda consistió en aplicar el instrumento por medio de una computadora (figura 1) permitiendo así el almacenamiento de los datos sin previa codificación, con mayor precisión y evitando errores.

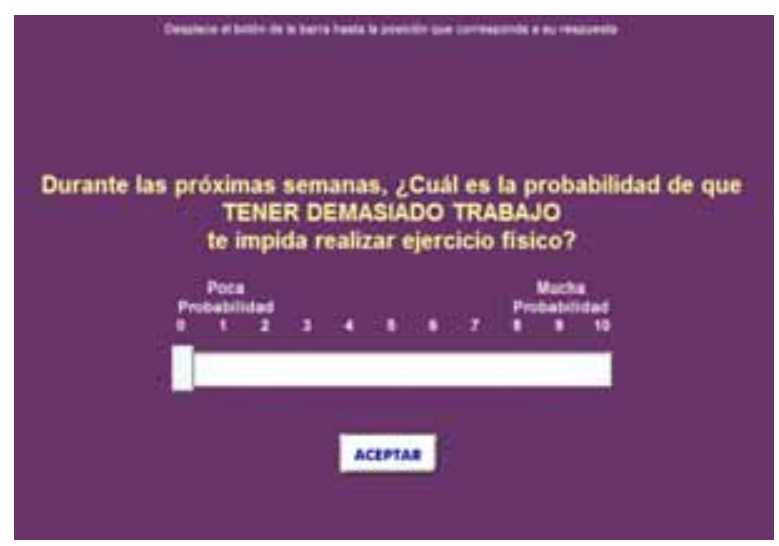

Figura 1. Ejemplo de respuesta para los ítems del cuestionario.

\section{Diseño}

En cuanto al diseño del estudio, se utilizó un enfoque cuantitativo con un diseño descriptivo y transversal tipo encuesta (Hernández, Fernández, \& Baptista, 2014). La variable independiente fue el género y las variables dependientes el promedio de las puntuaciones obtenidas en cada una de las subescalas del cuestionario ABPEF.

\section{Procedimiento}

Se invitó a participar en el estudio a los alumnos de las licenciaturas que se ofrecen en la Facultad de Ciencias de la Cultura Física de la UniversidadAutónoma de Chihuahua, México.

Los que aceptaron participar firmaron la carta de aceptación correspondiente. Luego se aplicó el instrumento, antes descrito, por medio de una computadora personal (módulo administrador del instrumento del editor de escalas de ejecución típica), en una sesión de aproximadamente 20 minutos; en los laboratorios o centros de cómputo de la Facultad de Ciencias de la Cultura Física.

Al inicio de cada sesión se hizo una pequeña introducción sobre la importancia de la investigación y de cómo acceder al instrumento. Se les solicitó la máxima sinceridad y se les garantizó la confidencialidad de los datos que se obtuvieran. Las instrucciones de cómo responder se encontraban en las primeras pantallas; antes del primer reactivo del instrumento. Al término de la sesión se les agradeció su participación.

Una vez aplicado el instrumento se procedió a recopilar los resultados por medio del módulo generador de resultados del editor de escalas versión 2.0 (Blanco et al., 2013).

\section{Análisis estadístico de los datos}

Se realizaron análisis estadísticos descriptivos (medias y desviaciones estándar) para todas las variables dependientes. Posteriormente, después de verificar que los datos cumplían con los supuestos de los análisis estadísticos paramétricos, se llevó a cabo un análisis multivariante de varianza(MANOVA), seguido por los análisis de varianza univariados (ANOVAs), para examinar las diferencias entre mujeres y hombres en cuanto a su percepción de barreras para la práctica de ejercicio físico, el tamaño del efecto se estimó mediante el eta cuadrado $\left(\eta^{2}\right)$. Todos los análisis estadísticos se realizaron con el programa SPSS versión 21.0 para Windows. El nivel de significación estadística se estableció en $p=.05$.

\section{Resultados}

La Tabla 1 muestra los valores de las medias y las desviaciones estándar de las puntuaciones de los cuatro factores estudiados sobre la percepción de barreras para la práctica de ejercicio físico, así como los resultados del MANOVA y los subsiguientes ANOVAs. Los resultados del MANOVA mostraron diferencias globales estadísticamente significativas de acuerdo a la variable género en las puntuaciones promedio de los factores del Autoinforme de Barreras para la Práctica de Ejercicio Físico (Wilks' $\lambda=.884 ; p<.001 ; \eta^{2}=.116$ ). Posteriormente, los resultados de los ANOVAs mostraron que las mujeres reportan mayor presencia de barreras en las cuatro dimensiones estudiadas; ansiedad física y social $(F=40.281, p<.001)$, fatiga o pereza $(F=94.004$, $p<.001)$, obligaciones y falta de tiempo $(F=66.774, p<.001) \mathrm{e}$ instalaciones $(F=20.925, p<.01)$.

Tabla 1.

Resultados MANOVA $y$ ANOVAs para las diferencias en percepción de barreras para la

\begin{tabular}{lccccc}
\hline $\begin{array}{l}\text { práctica de ejercicio físico de acuerdo al género. } \\
\text { mujeres } \\
(n=490)\end{array}$ & $\begin{array}{c}\text { hombres } \\
(n=407)\end{array}$ & $F$ & $p$ & $\eta^{2}$ \\
\hline & & & 29.210 & $<.001$ & .116 \\
Ansiedad física y social & $1.99(2.27)$ & $1.13(1.66)$ & 40.281 & $<.001$ & .043 \\
Fatiga o pereza & $3.67(1.89)$ & $2.48(1.76)$ & 94.004 & $<.001$ & .095 \\
Obligaciones y falta de tiempo & $4.82(2.47)$ & $3.47(2.47)$ & 66.774 & $<.001$ & .069 \\
Instalaciones & $2.27(1.97)$ & $1.69(1.80)$ & 20.925 & $<.01$ & .023 \\
\hline \multicolumn{2}{l}{ Nota. Los valores descriptivos se presentan como media (desviación estándar) }
\end{tabular}

\section{Discusión}

Los resultados indican que las mujeres son quienes reportan mayor presencia de barreras para la práctica de actividad física; lo que concuer- 
da con lo reportado en otras investigaciones donde se afirma que las mujeres, en comparación con hombres de la misma edad y nivel de estudios, perciben en mayor medida, que la falta de tiempo, la pereza o el estar cansadas les impiden realizar actividad física (Arboleda et al., 2016; Lovell et al., 2010; Serra et al., 2010). En un estudio realizado sobre los motivos para la práctica físico deportiva entre mujeres practicantes y no practicantes se encontró que en ambos grupos la salud es la razón más importante para practicar actividad física, mientras que para las mujeres que no practican el siguiente motivo fue la imagen, las chicas que si practican actividad física mostraron niveles más altos en desarrollo de la habilidad física, siendo el motivo más bajo el reconocimiento social en ambas muestras, sin embargo en el motivo de afiliación social se encontró diferencia entre las mujeres que practican y las que no, donde las no practicantes de actividad física consideran la afiliación social más importante (Moreno-Murcia, Pardo, Jorge, \& Huéscar, 2016). Por otro lado, Gil-Madrona, Valdivia-Moral, González-Víllora, y Zagalaz-Sánchez (2017) afirman que existe una sutil barrera por discriminación de género en la práctica de ejercicio físico en el tiempo de ocio, encontrando que los chicos hacen a un lado a las chicas cuando juegan con ellas, que las chicas se mofan de ellos profundizando el fracaso de los chicos, y que los chicos rara vez piensan que ellas son más débiles, sin embargo los estereotipos de género se van modificando a lo largo del crecimiento del niño; por tanto sería interesante indagar más al respecto.

\section{Conclusiones}

De los resultados mostrados, de su análisis y de su discusión, se pueden obtener las siguientes conclusiones:

1. Las mujeres en relación a los hombres perciben mayor probabilidad en la presencia de barreras para la práctica de ejercicio físico, dificultándoles la práctica de actividad física en mayor medida que los hombres.

2. Las diferencias encontradas entre hombres y mujeres en cuanto a la percepción de barreras para la práctica de ejercicio físico, sugieren además que al diseñar cualquier tipo de intervención que tenga como objetivo la disminución de la percepción de las mismas habrá que tomar en cuenta a la variable género; no obstante, es preciso desarrollar más investigación al respecto pues el tema trasciende del todo los alcances de la presente investigación. Se subraya además la importancia de realizar un mayor número de investigaciones sobre el tema en nuestro país.

Por último, cuando menos tres limitaciones están presentes en este trabajo. La primera es que los participantes son solo estudiantes universitarios mexicanos, lo que supone una amenaza para la posibilidad de generalizar estos resultados. Ampliar la muestra (agregando por ejemplo adultos jóvenes que no son estudiantes) es un área de trabajo de cara al futuro. La segunda limitación proviene del propio instrumento de medición, que se basa en el autoinforme y que por ello puede contener los sesgos que se derivan de la deseabilidad social. La tercera limitación es que no se han estudiado variables psicosociales en la muestra evaluada tales como características de la familia, nivel socioeconómico, deportes practicados, etc., o variables antropométricas, por lo que conviene desarrollar más investigación al respecto incluyendo dichas variables.

\section{Agradecimientos}

Este estudio es parte de un proyecto financiado por la Secretaría de Educación Pública-Subsecretaría de Educación Superior-Dirección de Superación Académica- Programa para el Desarrollo Profesional Docente (DE-13 -6894).

\section{Referencias}

Arboleda, V. H., Arango, E. F., \& Feito, Y. (2016). Actividad física y percepciones de beneficios y barreras en una universidad colombiana. RETOS. Nuevas tendencias en Educación Física, Deporte y Recreación(30), 15-19.

Blanco, H., Ornelas, M., Tristán, J. L., Cocca, A., Mayorga-Vega, D., LópezWalle, J., \& Viciana, J. (2013). Editor for creating and applying computerise surveys. Procedia Social and Behavioral Sciences, 106, 935-940. doi:10.1016/ j.sbspro.2013.12.105

El-Gilany, A. H., Badawi, K., El-Khawaga, G., \& Awadalla, N. (2011). Physical activity profile of students in Mansoura University, Egypt. Eastern MediterraneanHealth Journal, 17(8), 694-702.

ENSANUT. (2012). Encuesta Nacional de Salud y Nutrición 2012. Resultados nacionales.

Fisher, A., Wardle, J., Beeken, R. J., Croker, H., Williams, K., \& Grimmett, C. (2016). Perceived barriers and benefits to physical activity in colorectal cancer patients. Support Care Cancer, 24(2), 903-910. doi:10.1007/s00520-0152860-0

Gil-Madrona, P., Valdivia-Moral, P., González-Víllora, S., \& Zagalaz-Sánchez, M. L. (2017). Percepciones y comportamientos de discriminación sexual en la práctica de ejercicio físico entre los hombres y mujeres pre-adolescentes en el tiempo de ocio. Revista de Psicología del Deporte, 26(2), 0081-0086.

Gómez-López, M., Gallegos, A. G., \& Extremera, A. B. (2010). Perceived Barriers by University Students in the Practice of Physical Activities. Journal of Sports Science and Medicine, 9(3), 374-381.

Gutiérrez, J. P., Rivera-Dommarco, J., Shamah-Levy, T., Villalpando-Hernández, S., Franco, A., Cuevas-Nasu, L., . . . Hernández-Ávila, M. (2012). Encuesta Nacional de Salud y Nutrición 2012. Resultados Nacionales. Cuernavaca, México: Instituto Nacional de Salud Pública.

Hernández, R., Fernández, C., \& Baptista, P. (2014). Metodología de la investigación. México: McGraw- Hill.

Juarbe, T., Turok, X. P., \& Perez-Stable, E. J. (2002). Perceived Benefits and Barriers to Physical Activity among Older Latin Women. Western Journal of Nursing Research, 24(8), 868-886.

Leone, L. A., \& Ward, D. S. (2013). A Mixed Methods Comparison of Perceived Benefits and Barriers to Exercise between Obese and Non-Obese Women. Journal of Physical Activity and Health, 10(4), 461-469.

Lovell, G. P., Ansari, W. E. l., \& Parker, J. K. (2010). Perceived Exercise Benefits and Barriers of Non-Exercising Female University Students in the United Kingdom. International Journal of Environmental Research and Public Health, 7(3), 784-798. doi:10.3390/ijerph7030784

Meneses, M., \& Ruiz, F. (2017). Estudio longitudinal de los comportamientos y el nivel de actividad físico-deportiva en el tiempo libre en estudiantes de Costa Rica, México y España (Longitudinal study on leisure time behaviors and physical and sports activity level in students from. Retos: Nuevas tendencias en Educación Física, deporte y recreación (31), 219-226.

Meza-Peña, C., \& Pompa-Guajardo, E. G. (2016). Género, obesidad y autoconcepto en una muestra de adolescentes de México.[Gender, obesity and self concept in a sample of Mexican adolescents]. RICYDE. Revista Internacional de Ciencias del Deporte, 12(44), 137-148. doi:10. 5232/ ricyde2016. 04404

Moreno-Murcia, J. A., Pardo, M., Jorge, P., \& Huéscar, E. (2016). Motivos de práctica físico-deportiva en mujeres: Diferencias entre practicantes y no practicantes. Revista de Psicología del Deporte, 25(1), 0035-0041.

Muñoz-Daw, M. d. J., Muñoz-Duarte, M., De La Torre-Díaz, M. d. L., HinojosSeáñez, E., \& Pardo-Rentería, J. B. (2016). Motivos para la Práctica de Actividad Física Recreativa e Inactividad en la Población de Chihuahua (México). Nutrición clínica y Dietética Hospitalaria, 36(1), 10-16. doi:10.12873/ 361muñozdaw

Niñerola, J., Capdevila, L., \& Pintanel, M. (2006). Barreras percibidas y actividad física: el autoinforme de barreras para la práctica de ejercicio físico. Revista de Psicología del Deporte, 15(1), 53-69.

OMS. (2018). Actividad física. Recuperado de http://www.who.int/es/news-room/ fact-sheets/detail/physical-activity

Paredes, R., Orraca, O., Marimón, E. R., Casanova, M. d. l. C., \& González, L. M. (2014). Influencia del sedentarismo y la dieta Inadecuada en la salud de la población pinareña. Ciencias Médicas de Pinar del Río, 18(2), 221-230.

Patiño-Villada, F. A., Arango-Vélez, E. F., Quintero-Velásquez, M. A., \& ArenasSosa, M. M. (2011). Factores de riesgo cardiovascular en una población urbana de Colombia. Revista de Salud Pública, 13(3), 433-445.

Pavón, A., \& Moreno, J. A. (2008). Actitud de los universitarios ante la práctica físico deportiva: Diferencias por géneros. Revista de Psicología del Deporte, 17(1), 7-23.

Ramírez-Vélez, R., Tordecilla-Sanders, A., Laverde, D., Hernández-Novoa, J. G., Ríos, M., Rubio, F., . . . Martínez-Torres, J. (2015). The prevalence of barriers for Colombian college students engaging in physical activity. Nutrición Hospitalaria, 31(2), 858-865. doi:10.3305/nh.2015.31.2.7737

Ramírez-Vélez, R., Triana-Reina, H. R., Carrillo, H. A., \& Ramos-Sepúlveda, J. A. (2016). Percepción de barreras para la práctica de la actividad física y obesidad abdominal en universitarios de Colombia. Nutrición Hospitalaria, 33(6), 1317-1323. doi:10.20960/nh.777

Serra, J. R., Generelo, E., \& Zaragoza, J. (2010). Barreras para la realización de actividad física en adolescentes en la provincia de Huesca. Revista Internacional de Medicina y Ciencias de la Actividad Física y el Deporte, 10(39), 470-482.

Stankov, I., Olds, T., \& Cargo, M. (2012). Overweight and obese adolescents: what turns them off physical activity? International Journal of Behavioral Nutrition and Physical Activity, 9(53), 1-15. doi:10.1186/1479-5868-9-53

Velasco-Contreras, M. E. (2013). Perfil de salud de los trabajadores del Instituto Mexicano del Seguro Social. Revista Médica del Instituto Mexicano del Seguro Social, 51(1), 12-25.

Vélez-Álvarez, C., Vidarte-Claros, J. A., \& Parra-Sánchez, J. H. (2014). Niveles de sedentarismo en población entre 18 y 60 años en Manizales, Pereira y Armenia, Colombia. Análisis Multivariado. Aquichán, 14(3), 303-315 doi:10.5294/ aqui.2014.14.3.3 